RUNNING HEAD: REGULARITIES IN LEARNING

\title{
Environmental Regularities as a Concept for Carving up the Realm of Learning Research: Implications for Relational Frame Theory
}

\author{
Jan De Houwer \& Sean Hughes \\ Ghent University, Ghent, Belgium
}

In Press. Journal of Contextual Behavioral Science

\author{
mailing address: Jan De Houwer \\ Ghent University \\ Henri Dunantlaan 2 \\ B-9000 Ghent \\ Belgium \\ email: Jan.DeHouwer@UGent.be \\ phone: 003292646445 \\ fax: 003292646489
}




\begin{abstract}
Learning can be defined functionally as the impact of regularities in the environment on behavior. The concept of environmental regularities is a crucial part of this definition because it (a) improves the scope and depth of the definition and (b) provides ways to differentiate between different types of learning. We argue that this concept is useful also for conceptualizing learning from the perspective of Relational Frame Theory. More specifically, even if all instances of learning qualify as instances of Arbitrarily Applicable Relational Responding, different types of learning could still be functionally different because they involve different types of proximal regularities.
\end{abstract}

Keywords: environmental regularities, learning, Relational Frame Theory 


\section{Environmental Regularities as a Concept for Carving up the Realm of Learning Research: Implications for Relational Frame Theory}

Although learning is a core concept in psychology, opinions differ about what it means to say that learning has occurred (see Barron et al., 2015, for a recent review). In this paper, we revisit and extend the work of De Houwer, Barnes-Holmes, and Moors (2013) who argued that learning can be conceived of as the impact of regularities in the environment on behavior. More specifically, we explore the merits of the concept environmental regularity not only for the definition of learning but also as a way of carving up the realm of learning research. We argue that this concept is useful not only for an analysis of learning that predates the development of Relational Frame Theory (RFT) (i.e., Learning 1.0) but also for an analysis of learning as conceived of from the perspective of RFT (i.e., Learning 2.0). ${ }^{1}$

\section{Learning 1.0: The Impact of Environmental Regularities on Behavior}

One way to evaluate the utility of scientific terms and concepts is to determine the extent to which they allow researchers to operate with precision, scope, and depth (see Hayes, Barnes-Holmes, \& Wilson, 2012; Wilson, 2015). Take, for instance, the definition of learning. Before the paper of De Houwer et al. (2013) learning had been defined functionally as the impact of the environment on behavior and mentally as the storage of information in memory (e.g., see Barron et al., 2015; Lachman, 1997). Yet these definitions are problematic for different reasons. Most importantly, the scope of the functional definition is too broad because it includes all effects of the environment on behavior, even those that do not seem to qualify as instances of learning (e.g., behavior due to an individual stimulus that is present at a particular point in time such as a startle response that is evoked by the presentation of a loud bang). One

\footnotetext{
1 Our use of the terms Learning 1.0 and Learning 2.0 is not meant to imply that the development of RFT caused a fundamental break in the history of learning research. It is quite clear that RFT itself draws on traditional concepts such as the concept of a generalized operant. Our distinction merely implies that RFT offers a fundamentally different perspective on many learning phenomena.
} 
could also argue that the scope of the mental definition of learning is too narrow because it only allows for learning in entities that have a memory system and that are thus able to store information. The mental definition has the additional disadvantage that it is difficult to verify because it requires the verification of something non-physical (information in memory) which brings with it a host of problems (De Houwer, 2011).

Based on these and other considerations, De Houwer et al. (2013) opted for a new functional definition of learning, one that improved the scope and depth of previous definitions. They achieved this by introducing the concept of environmental regularity which they defined as "all states in the environment of the organism that entail more than the presence of a single stimulus or behavior at a single moment in time. It can thus refer to the presence of a single stimulus or behavior at multiple moments in time, the presence of multiple stimuli or behaviors at a single moment in time (as in one-trial learning), and the presence of multiple stimuli or behaviors at multiple moments in time” (p. 634). Using this concept, learning was defined as "changes in the behavior of an organism that are the result of regularities in the environment of that organism” (p. 633). ${ }^{2}$

Introducing the concept of environmental regularities has several advantages. First, it improves the scope of the definition as compared to earlier functional definitions of learning by allowing one to exclude changes in behavior that are due to the elements in the environment but that do not qualify as instances of learning (e.g., behavior due to a single stimulus at a single moment in time, such as a startle response to a loud bang). Second, it improves depth by increasing the extent to which the definition of learning coheres with scientific activity at other levels of explanation (Hayes et al., 2012). For instance, at the supra-individual level, De

2 Although a regularity as defined by De Houwer et al. (2013) can occur at a single moment in time, establishing learning always requires the consideration of events that occur at different points in time. For instance, to establish one-trial Pavlovian conditioning, it needs to be verified whether the reaction to the CS at Time 2 changes as the result of a single CS-US pairing at Time 1. 
Houwer et al.’s definition of learning sheds new light on the relationship between learning and evolution. More specifically, it highlights that learning and evolution both deal with adaptation, that is, the way in which regularities in the environment influence the behavior of organisms. Whereas learning refers to ontogenetic adaptation (i.e., adaptation of a single organism during the lifetime of that individual; also see Skinner, 1938, 1984), evolution refers to phylogenetic adaptation (i.e., adaptation of a group of organisms across the lifetime of different organisms). At the sub-individual level, De Houwer et al.'s definition of learning is compatible with the idea that individual parts of organisms, such as neurons, cell assemblies, or genetic material may also adapt to regularities in the environment. For instance, in light of evidence from the field of epigenetics research showing that the activity of genetic material depends on environmental events (e.g., Carey, 2013), it would be interesting to examine how this activity changes as the result of environmental regularities. ${ }^{3}$ In sum, although psychologists are typically interested in explanations at the level of the whole organism, defining learning in terms of adaptation allows them to interact in productive ways with scientists who study adaptation at other levels of explanation (Hayes et al., 2012).

Another major advantage of the concept of environmental regularities is that it allows one to carve up the realm of learning research. Although it is good to have concepts that have scope and depth, researchers sometimes also need to make distinctions in order to increase precision. Introducing the concept of environmental regularities not only allows one to determine what qualifies as an instance of learning and what does not, it also provides a potentially useful way of differentiating between different types of learning. More specifically, De Houwer et al. (2013) defined different types of learning (i.e., non-associative learning, classical conditioning, and operant conditioning) as the effect of different types of 
environmental regularities (i.e., regularities in the presence of a single stimulus, regularities in the presence of two or more stimuli, and regularities in the presence of stimuli and behavior, respectively). As such, the concept of environmental regularities allows for a taxonomy of learning effects. Within this taxonomy, further distinctions can be made on the basis of additional criteria, for instance, the type of behavior that changes (e.g., evaluative conditioning as the impact of stimulus pairings on evaluative behavior) or the direction of change (e.g., habituation as an decrease in responding versus sensitization as an increase in responding as a function of repeated stimulus presentations).

Taxonomies not only allow one to bring order to what is already known (i.e., have heuristic value) but also to delineate that which is not yet known (i.e., have predictive value). For instance, inspired by the taxonomy of De Houwer et al. (2013), Hughes, De Houwer, and Perugini (2016) recently proposed that intersections between different environmental regularities can also lead to changes in behavior. To illustrate, consider a task in which participants are asked to press a left key whenever they see a positive stimulus or a first novel brand name and to press a right key whenever they see a negative image or a second novel brand name. This situation involves four environmental regularities, more specifically, four operant contingencies (i.e., Positive: Left - Correct; Novel Brand 1: Left - Correct; Negative: Right - Correct; Novel Brand 2: Right - Correct). Importantly, the first two contingencies intersect in that they both involve a left response whereas the other two contingencies intersect in that they both involve a right response. After performing this task, participants typically like the first novel brand name more than the second one (also see Prestwich, Perugini, Hurling, \& Richetin, 2010). Hughes et al. argued that this might just be one instance of a larger class of learning effects in which evaluative behavior changes as the result of intersections between regularities in the environment. In support of this idea, they demonstrated that also other types 
of intersections between regularities can lead to changes in liking. Moreover, several known learning phenomena (e.g., the differential outcomes effect; see Zentall, Wasserman, \& Urcuioli, 2014) can also be conceptualized as instances of learning via intersecting regularities. ${ }^{4}$ Thus the idea of intersecting regularities dramatically increases the range of learning phenomena that can be captured and predicted on the basis of a taxonomy of learning that is built on the concept of environmental regularities. It also illustrates how this concept can inspire new developments in learning research.

\section{Learning 2.0: What Role Can the Concept of Environmental Regularities Play in RFT?}

RFT suggests that many, if not all instances of learning (at least in verbal humans) involve some element of arbitrarily applicable relational responding (AARR; Hayes et al., 2001). If one takes this claim seriously then the concept of AARR is quite simply a 'game changer' for theory and research on learning because it requires us to rethink much of what we originally assumed about learning. In our opinion, the concept of environmental regularities has implications also for learning as conceptualized within RFT. More specifically, we believe that environmental regularities can be used to clarify not only to clarify what it means to say that instances of learning are instances of AARR but also to distinguish between different types of learning that are all instances of AARR. To understand our position, it is first important to realize that the effects of different environmental regularities can interact. De Houwer et al. (2013) introduced the concept of moderated learning to refer to changes in behavior that are due to a particular environmental regularity but in a way that is moderated by other

\footnotetext{
4 Note that learning via intersecting regularities is not the same as class intersections (or class unions; Sidman, 1994). Class intersections (or unions) refer to certain patterns of behavior that can emerge as the result of various types of environmental regularities. Learning via intersecting regularities, on the other hand, is defined in terms of the specific type of environmental regularity that is responsible for the change in behavior rather than the nature of the change in behavior. Although some instances of learning might qualify both as learning via intersecting regularities and as class intersections (or unions), there might be instances of class intersections (or unions) that are not due to intersecting regularities or changes in behavior that are due to intersecting regularities but that do not have the properties of class intersections (or unions).
} 
environmental regularities. For instance, in sensory preconditioning, the impact of stimulus pairings on behavior (e.g., the impact of tone-light pairings on fear responses to the tone) depends heavily on the presence of other stimulus pairings (e.g., subsequent pairings of the light and a shock). As De Houwer et al. noted:

Moderated learning effects are important because they demonstrate that the causal effect of regularities on behavior should always be considered in a broader context of other regularities. It also has an important adaptive value in that it allows for a fine tuning of learning itself. More specifically, moderated learning can be conceived of as "ontogenetic adaptation of learning" or "learning of learning"-that is, effects of regularities in the environment on how other regularities in the environment influence behavior. As such, moderated learning dramatically increases the flexibility with which organisms can adapt to their environment. (p. 642)

The concept of moderated learning helps us to clarify what it means to say that an instance of learning is an instance of AARR. RFT postulates that AARR critically depends on a specific learning history that all verbal beings have experienced (Hayes, Barnes-Holmes, \& Roche, 2001). Importantly, this learning history can be conceptualized as involving an extensive set of environmental regularities. From this perspective, the claim that an instance of learning is an instance of AARR implies that learning is moderated by the distal regularities that give rise to AARR. Hence, learning as AARR qualifies as moderated learning. Linking the concept of environmental regularities to RFT in this manner also allows us to retain the idea that different types of learning involve different types of environmental regularities. Although AARR always involves a specific set of distal regularities (i.e., those involved in the learning history that gives rise to AARR), these distal regularities can moderate the effect of various types of proximal regularities (i.e., regularities in the current environment, such as the pairing 
of stimuli). Hence, one can still distinguish different types of learning on the basis of the nature of the proximal regularity that is involved, even when all types of learning are thought to be instances of AARR. ${ }^{5}$

We believe that it is not only possible but also useful for RFT researchers to distinguish between different types of learning in this way. First, it allows RFT researchers to clarify how their perspective on learning differs from other perspectives (i.e., that learning involves not only proximal regularities but also a specific set of distal regularities that moderate the impact of the proximal regularities). Second, it highlights the possibility that instances of AARR that involve different proximal regularities differ in their respective functional properties. So far, the majority of RFT research has focused on a single type of procedure (Matching-to-Sample; MTS) which is itself based on a single type of environmental regularity (i.e., behavior-consequence relations). Although some studies have used other proximal regularities (e.g., stimulus-stimulus pairings; Leader, Barnes, \& Smeets, 1996) little is known about whether the type of proximal regularity determines the properties of AARR. Introducing the concept of (proximal) environmental regularities in RFT research underscores the potential moderating role of the nature of these regularities. Put differently, RFT research has focused primarily on the nature of the distal regularities that transform humans into verbal beings and the way in which these distal proximal moderate the impact of proximal regularities on behavior. We argue that there might be additional merit in examining how the nature of the proximal regularities moderates the moderating impact of the distal regularities. That is, the precise way in which distal regularities moderate the impact of proximal regularities might

\footnotetext{
5 Note that RFT contains many other concepts that allow one to draw distinctions and thus talk in a precise way about learning (e.g., different instances of AARR as involving different relational frames or different types of contextual cues). Our point is that the concept of environmental regularities can offer an additional conceptual tool to RFT researchers for increasing precision.
} 
depend on the nature of the proximal regularities themselves. In the remainder of this section, we will illustrate these ideas in the context of evaluative learning research.

We have increasingly adopted this enriched RFT perspective in our own work on evaluative conditioning (e.g., De Houwer \& Hughes, in press; Hughes, De Houwer, \& Barnes-Holmes, 2016). Evaluative conditioning refers to a change in liking that occurs due to the pairing of stimuli (De Houwer, 2007). For instance, after repeatedly presenting a neutral face with positive pictures and another neutral face with negative pictures, the first face will typically be liked more than the second (Levey \& Martin, 1975). Most researchers think of evaluative conditioning as a subclass of classical conditioning effects, namely those effects that involve changes in evaluative (rather than non-evaluative) behavior. From this perspective, stimulus pairings function as a proximal cause of the change in liking, that is, as an environmental regularity in the current context that influences evaluative behavior. Yet from an RFT perspective, stimulus pairings may function as a contextual cue signaling how those stimuli can be related. For instance, the fact that stimuli are presented together in space and time could cue people to treat them as being similar to one another. In the context of evaluative conditioning, this may include similarity in terms of evaluative responses. This view implies that evaluative conditioning effects are actually instances of AARR in which one stimulus (CS) is related to another (US) under the control of a contextual cue (pairings), and as a result, a change in liking (transformation of evaluative functions) takes place.

Despite the potential added value of RFT for evaluative conditioning research (see Hughes et al., 2016), viewing evaluative conditioning as an instance of AARR might be seen as blurring the distinction between evaluative conditioning and other types of (evaluative) learning (De Houwer \& Hughes, in press). For instance, given their reliance on language, changes in liking that result from verbal persuasive messages are also likely to be instances of 
AARR. One could even argue that mere exposure effects (i.e., changes in liking as the result of the repeated presentation of a single stimulus) qualify as instances of AARR. For instance, the repeated presentation of a stimulus may function as a relational cue signaling that the presented stimulus is similar to other stimuli that the individual likes. Repeated stimulus presentations might have acquired that function because liked stimuli tend to occur more frequently than disliked stimuli. However, by incorporating the concept of environmental regularities into our RFT approach, we could benefit from the added value of RFT while maintaining the distinction between different types of evaluative learning. Different types of evaluative learning might be fundamentally similar (i.e., all types are instances of AARR) but still unique with regard to the proximal regularity involved (e.g., repeated stimulus presentations in mere exposure and stimulus pairings in evaluative conditioning) and therefore potentially unique in the conditions under which liking can be changed. Assume, for instance, that an advertiser would like to increase consumers' liking of a novel brand. (S)he can do this by pairing the brand with positive images (as in evaluative conditioning) or by informing customers that the novel brand has positive properties (as in persuasion). Even though both approaches may give rise to instances of AARR (i.e., both the pairings and the persuasive message may function as cues for specific patterns of relational responding), it could be that only the persuasive message leads to reactance effects (e.g., because the consumers feel coerced into liking the novel brand). Regardless of whether empirical research confirms this hypothesis, there is merit in exploring the ways in which the nature of the proximal regularity that gives rise to changes in liking moderates evaluative learning, even when all instances of evaluative learning qualify as instances of AARR. 


\section{Conclusion}

In this paper, we argued that the concept of environmental regularities can provide an potentially useful tool for learning research, including RFT inspired research. It not only reveals what different learning phenomena have in common but also how they differ. Although environmental regularities refer only to spatio-temporal properties of events (e.g., the fact that two stimuli repeatedly co-occur in space and time), they might also help researchers to discover interesting functional knowledge (e.g., that the properties of AARR depend on the nature of the proximal regularity). We therefore hope that researchers will embrace the concept and explore its potential. 


\section{References}

Barnes-Holmes, D., \& Hussey, I. (2016). The functional-cognitive meta-theoretical framework: Reflections, possible clarifications and how to move forward. International Journal of Psychology, 51, 50-57.

Barron, A. B., Hebets, E. A., Cleland, T. A., Fitzpatrick, C.L., Hauber, M. E., and Stevens, J. R. (2015). Embracing multiple definitions of learning. Trends in Cognitive Science, 38, 405-407.

Carey, N. (2013). The Epigenetics Revolution. NY: Columbia University Press.

De Houwer, J. (2007). A conceptual and theoretical analysis of EC. The Spanish Journal of Psychology, 10, 230-241.

De Houwer, J. (2011). Why the cognitive approach in psychology would profit from a functional approach and vice versa. Perspectives on Psychological Science, 6, 202-209.

De Houwer, J., Barnes-Holmes, D., \& Moors, A. (2013). What is learning? On the nature and merits of a functional definition of learning. Psychonomic Bulletin \& Review, 20, 631-642.

De Houwer, J., \& Hughes, S. (in press). Evaluative conditioning as a symbolic phenomenon: On the relation between evaluative conditioning, evaluative conditioning via instructions, and persuasion. Social Cognition.

Hayes, S. C., Barnes-Holmes, D., \& Roche, B. (Eds.). (2001). Relational Frame Theory: A Post-Skinnerian account of human language and cognition. New York: Plenum Press.

Hayes, S. C., Barnes-Holmes, D., \& Wilson, K. (2012). Contextual behavioral science: Creating a science more adequate to the challenge of the human condition. Journal of Contextual Behavioral Science, 1, 1-16.

Hughes, S., De Houwer, J., \& Barnes-Holmes, D. (2016). The moderating impact of distal 
regularities on the effect of stimulus pairings: A novel perspective on evaluative conditioning. Experimental Psychology, 63, 20-44.

Hughes, S., De Houwer, J., \& Perugini, M. (2016). Expanding the boundaries of evaluative learning research: How intersecting regularities shape our likes and dislikes. Journal of Experimental Psychology: General, 145, 731-754.

Hughes, S., \& Barnes-Holmes, D. (2016). Relational frame theory: Implications for the study of human language and cognition. In R. D. Zettle, S. C. Hayes, D. Barnes-Holmes, \& A. Biglan (Eds), The Wiley handbook of contextual behavioral science (pp. 179-226), West Sussex, UK: Wiley-Blackwell.

Lachman, S. J. (1997). Learning is a process: Toward an improved definition of learning. Journal of Psychology, 131, 477-480.

Leader, G., Barnes, D., \& Smeets, P. M. (1996). Establishing equivalence relations using a respondent-type training procedure. The Psychological Record, 46(4), 685-706.

Levey, A. B., \& Martin, I. (1975). Classical conditioning of human evaluative responses. Behaviour Research and Therapy, 4, 205-207.

Prestwich, A., Perugini, M., Hurling, R., \& Richetin, J. (2010). Using the self to change implicit attitudes. European Journal of Social Psychology, 40, 61-71.

Sidman, M. (1994). Equivalence relations: A research story. Boston: Authors Cooperative.

Skinner, B.F. (1938). The Behavior of Organisms: An Experimental Analysis. New York: Appleton-Century.

Skinner, B. F. (1984). The evolution of behavior. Journal of the Experimental Analysis of Behavior, 41, 217-221.

Wilson, K. G. (2015). Contextual behavioral science: Holding terms lightly. In R. D. Zettle, S. C. Hayes, D. Barnes-Holmes, \& A. Biglan (Eds), The Wiley Handbook of Contextual 
Behavioral Science (pp. 62-79). West Sussex, UK: Wiley-Blackwell..

Zentall, T. R., Wasserman, E. A., \& Urcuioli, P. J. (2014). Associative concept learning in animals. Journal of the Experimental Analysis of Behavior, 101, 130-151. 


\section{Author Note}

Jan De Houwer and Sean Hughes, Ghent University, Belgium. The preparation of this paper was made possible by Grant BOF16/MET_V/002 of Ghent University and the Belgian Science Policy under the Interuniversity Attraction Poles programme (IAP7/33). We thank Dermot Barnes-Holmes for the numerous discussions on topics related to this paper.

Correspondence should be addressed to Jan De Houwer, Ghent University, Henri Dunantlaan 2, B-9000 Ghent, Belgium. Electronic mail can be sent to Jan.DeHouwer@UGent.be . 